NON-FAILURE OF FILAMENTS AND GLOBAL EXISTENCE FOR THE EQUATIONS OF FIBER SPINNING

By

Thomas Hagen

and

Michael Renardy

IMA Preprint Series \# 2320

(June 2010)

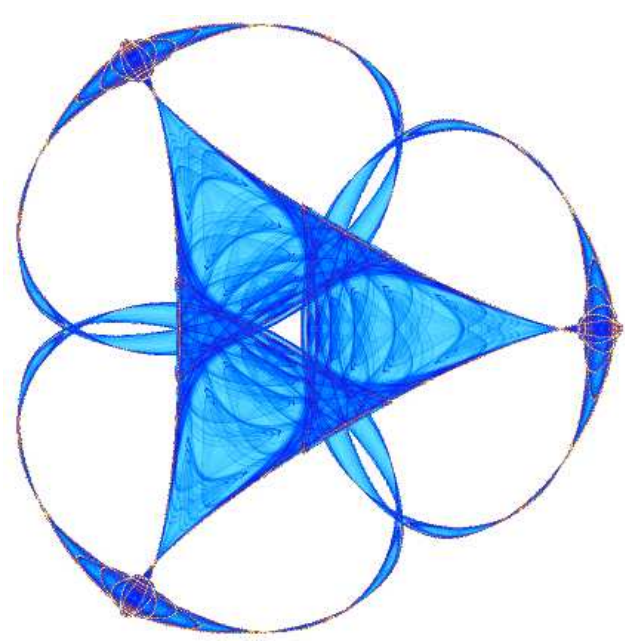

INSTITUTE FOR MATHEMATICS AND ITS APPLICATIONS

UNIVERSITY OF MINNESOTA 400 Lind Hall

207 Church Street S.E.

Minneapolis, Minnesota 55455-0436

Phone: 612-624-6066 Fax: 612-626-7370

URL: http://www.ima.umn.edu 


\title{
Non-Failure of Filaments and Global Existence for the Equations of Fiber Spinning
}

\author{
Thomas Hagen $^{1 *}$ and Michael Renardy ${ }^{2 \dagger}$ \\ ${ }^{1}$ Department of Mathematical Sciences, The University of Memphis, \\ Memphis, TN 38152, USA \\ ${ }^{2}$ Department of Mathematics, Virginia Polytechnic Institute and \\ State University, Blacksburg, Virginia 24061-0123, USA
}

May 2010

\begin{abstract}
In this work we give a global-in-time existence and uniqueness proof for solutions of the isothermal equations of fiber spinning. Fiber spinning is a widely used manufacturing process for the production of long thin filaments. In this process a highly viscous fluid is withdrawn from a reservoir and stretched to form a long fiber. The equations modeling fiber spinning are essentially based on cross-sectional averaging of the axisymmetric Stokes equations with free boundary. They form a coupled system consisting of a nonlinear mass transport equation and a nonlinear momentum conservation equation for dominant viscous forces in one space dimension.

Our analytical approach is based on a representation result of the fiber cross-sectional area in terms of a suitably chosen Lagrangian variable. This representation shows that viscous fibers do not break in finite time and that solutions retain their smoothness. These two results suffice to extend local-in-time solutions to global-in-time solutions. Our no-breakup result is in agreement with previous related work.
\end{abstract}

Key words and phrases: Thin filament approximation; Lagrangian variable; global existence of solutions; viscous fluid

\section{Introduction}

Fiber spinning (or fiber drawing) is an industrial application to produce long thin filaments. A highly viscous liquid such as a glass melt is withdrawn through a circular die and extended axially to form a long fiber which is wound up on a chilling spool and moved on to post-processing. The fiber spinning

*electronic mail: thagen@memphis.edu

†electronic mail: renardym@math.vt.edu 
process is known to exhibit flow instabilities limiting the production output and is therefore the object of intensive studies in engineering.

In the following we consider isothermal fiber spinning of a highly viscous liquid. The model for this flow assumes constant fluid viscosity and negligible inertia, gravity and surface tension. We denote time by $t$, the axial variable in the direction of stretching by $z$, the fiber cross-sectional area by $a=a(t, z)$, and the axial velocity by $v=v(t, z)$. The governing equations are given by the equation for mass conservation

$$
a_{t}+(v a)_{z}=0
$$

and the equation for momentum conservation

$$
\left(a v_{z}\right)_{z}=0
$$

Both equations are written in dimensionless form on the normalized domain $0 \leq z \leq 1, t \geq 0$. The fluid inlet and wind-up points are assumed at $z=0$ and $z=1$, respectively. To close the formulation of the problem one imposes boundary conditions of the form

$$
\begin{aligned}
& a(t, 0)=a_{0}(t), \\
& v(t, 0)=v_{0}(t), \\
& v(t, 1)=v_{1}(t)
\end{aligned}
$$

and an initial condition of the form

$$
a(0, z)=a_{\alpha}(z) .
$$

Throughout it is assumed that $a_{0}(t)>0, a_{\alpha}(t)>0$ and that $v_{1}(t)>v_{0}(t)>0$ for $t \geq 0$. The latter inequality reflects the requirement that the wind-up velocity be larger than the positive inflow velocity. Additional conditions guaranteeing the local existence of solutions will be formulated below. Subscript Latin letters denote partial derivatives. The subscript $\alpha$ is reserved for initial data. Total derivatives will typically be denoted by a prime with the exception of a total time derivative which we denote by a superscript dot. Derivatives in boundary points will be tacitly understood as one-sided derivatives.

The governing equations arise as the thin filament approximation of the axisymmetric Stokes equations with moving (free) boundary. For the purely viscous case discussed here these equations were essentially introduced by Kase and Matsuo [8]. Matovich and Pearson [9] gave a formal, yet systematic derivation of the governing equations from the (Navier-) Stokes equations by exploiting the smallness of the aspect ratio of radial versus axial length scale and thus reducing the underlying two-dimensional flow to one spatial dimension. For actual spinning applications this aspect ratio is $1: 100$ and can be as small as $1: 1000$.

In this work we analyze the global existence and uniqueness of solutions for the boundary-initial value problem given by Eqs. (1.1)-(1.6). To this end, we first review a known local-in-time existence and uniqueness result of solutions 
which will serve as a starting point for our global existence result. An important milestone in our approach consists in showing that the fiber radius for any (localin-time) solution stays positive and regularity of solutions is retained. Hence viscous filaments modeled by the equations of fiber spinning (1.1)-(1.6) do not fail in finite time. Finally we use this result to prove that local solutions extend to global solutions.

\section{Review: A Local Existence Result}

In this section we review a local existence result for the governing equations. The notions and results given were discussed in a series of works $[1,2,3,5,6]$ which contained additional technical details and extensions to more complicated fluid models.

Let $z_{1}<z_{2}, t^{\prime}>0$ and $m, n \in \mathbb{N}_{0}$. Throughout this section we will use the following norms:

- $\|\cdot\|_{p}$ for the norm on the Lebesgue space $L^{p}\left(z_{1}, z_{2}\right), 1 \leq p \leq \infty$,

- $\|\cdot\|_{H^{n}}$ for the norm on the Sobolev space $H^{n}\left(z_{1}, z_{2}\right)$,

- $\|\cdot\|_{m, n}$ for the norm on the Sobolev-Bochner space $W^{m, \infty}\left(\left[0, t^{\prime}\right] ; H^{n}\left(z_{1}, z_{2}\right)\right)$.

The idea underlying the following local existence/uniqueness proof for the governing equations is to obtain the solution as the fixed point of an appropriate operator. In particular, this solution operator takes as its input a candidate $b$ for the cross-sectional area and produces as its output the solution $\tilde{b}$ of the following linear advection problem

$$
\begin{aligned}
& \tilde{b}_{t}+w \tilde{b}_{z}+b w_{z}=0, \quad t \geq 0, \quad 0 \leq z \leq 1, \\
& \tilde{b}(t, 0)=a_{0}(t), \quad t \geq 0 \\
& \tilde{b}(0, z)=a_{\alpha}(z), \quad 0 \leq z \leq 1
\end{aligned}
$$

where

$$
w(t, z)=v_{0}(t)+\left(v_{1}(t)-v_{0}(t)\right) \frac{\int_{0}^{z}(b(t, x))^{-1} d x}{\int_{0}^{1}(b(t, x))^{-1} d x} .
$$

This expression for $w$ originates from solving Eq. (1.2) for $v$ and replacing $a$ and $v$ by $b$ and $w$, respectively.

The main step in the construction of the solution operator is to understand the general linear advection problem

$$
\begin{aligned}
& u_{t}(t, x)+p(t, x) u_{x}(t, x)=f(t, x), \quad t \geq 0, \quad 0 \leq x \leq 1, \\
& u(t, 0)=u_{0}(t), \quad t \geq 0, \\
& u(0, x)=u_{\alpha}(x), \quad 0 \leq x \leq 1
\end{aligned}
$$

for given $p, f, u_{0}$ and $u_{\alpha}$. To this end we make use of the notion of boundary regularity, introduced in $[1,5]$. 
Definition 2.1 The space $\mathbb{B} \mathbb{R}\left(0, t^{\prime} ; 0,1\right)$ of boundary-regular functions consists of all functions $g=g(t, x)$ on $\left[0, t^{\prime}\right] \times[0,1]$ such that

$$
\begin{aligned}
& g \in W^{1, \infty}\left(\left[0, t^{\prime}\right] ; H^{1}(0,1)\right) \cap L^{\infty}\left(\left[0, t^{\prime}\right] ; H^{2}(0,1)\right), \\
& g_{x}(\cdot, 0), g_{x}(\cdot, 1) \in H^{1}\left(0, t^{\prime}\right) .
\end{aligned}
$$

The space $\mathbb{B} \mathbb{R}\left(0, t^{\prime} ; 0,1\right)$ is endowed with the energy norm

$$
\mathcal{E}(g) \stackrel{\text { def }}{=}\left(\|g\|_{0,2}^{2}+\|g\|_{1,1}^{2}+\left\|g_{x}(\cdot, 0)\right\|_{H^{1}}^{2}+\left\|g_{x}(\cdot, 1)\right\|_{H^{1}}^{2}\right)^{\frac{1}{2}} .
$$

As is shown in $[1,5]$ boundary-regular functions can be approximated by $C^{\infty}$ functions to obtain suitable a priori estimates for the solution operator and as such form a technical tool for the relevant existence theory. We state the following important result from $[1,5]$ for further reference.

Lemma 2.2 Let $f, p, u^{0}$ and $u^{*}$ be functions such that

$$
\begin{aligned}
& p, f \in \mathbb{B} \mathbb{R}\left(0, t^{\prime} ; 0,1\right), \\
& p>0 \text { on }\left[0, t^{\prime}\right] \times[0,1], \\
& u_{0} \in H^{2}\left(0, t^{\prime}\right), \quad u_{\alpha} \in H^{2}(0,1), \\
& u_{0}(0)=u_{\alpha}(0), \\
& \dot{u}_{0}(0)+p(0,0) u_{\alpha}^{\prime}(0)=f(0,0) .
\end{aligned}
$$

Then the boundary-initial value problem (2.5)-(2.7) has a solution u such that

$$
\begin{aligned}
& u \in C^{1}\left(\left[0, t^{\prime}\right] ; H^{1}(0,1)\right) \cap C\left(\left[0, t^{\prime}\right] ; H^{2}(0,1)\right) \cap \mathbb{B R}\left(0, t^{\prime} ; 0,1\right), \\
& u \text { is unique in } W^{1, \infty}\left(\left[0, t^{\prime}\right] ; L^{2}(0,1)\right) \cap L^{\infty}\left(\left[0, t^{\prime}\right] ; H^{1}(0,1)\right) .
\end{aligned}
$$

Using the fixed-point strategy outlined above, we gave essentially the following local-in-time existence and uniqueness result for the equations of fiber spinning in [1]. A local existence proof for the equations of fiber spinning of a viscoelastic fluid with Newtonian stresses appeared in [3]. This latter work contains the problem discussed here as a special case. Local-in-time solvability of fiber spinning of a viscoelastic fluid without Newtonian stresses was recently addressed in [4].

Theorem 2.3 Suppose that

$$
\begin{aligned}
& a_{0} \in H^{2}\left(0, t^{\prime}\right), \quad a_{\alpha} \in H^{2}(0,1), \quad a_{0}>0, \quad a_{\alpha}>0, \\
& v_{0}, v_{1} \in C^{1}\left(\left[0, t^{\prime}\right]\right), \quad 0<v_{0}<v_{1}
\end{aligned}
$$

are given such that the compatibility conditions

$$
\begin{aligned}
& a_{0}(0)=a_{\alpha}(0), \\
& \dot{a}_{0}(0)+v_{0} a_{\alpha}^{\prime}(0)+\frac{v_{1}(0)-v_{0}(0)}{\int_{0}^{1} a_{\alpha}^{-1}(x) d x}=0
\end{aligned}
$$


hold. Then there exists $t_{0} \in\left(0, t^{\prime}\right]$ such that the boundary-initial value problem (1.1)-(1.6) has a unique solution $(a, v)$ on $\left[0, t_{0}\right] \times[0,1]$ with the properties

$$
\begin{aligned}
& a \in \bigcap_{k=0}^{2} C^{k}\left(\left[0, t_{0}\right] ; H^{2-k}(0,1)\right), \quad a>0, \\
& v \in C^{1}\left(\left[0, t_{0}\right] ; H^{1}(0,1)\right) \cap C\left(\left[0, t_{0}\right] ; H^{2}(0,1)\right), \\
& a, v \in \mathbb{B} \mathbb{R}\left(0, t_{0} ; 0,1\right) .
\end{aligned}
$$

Note that the solution $(a, v)$ given by Theorem 2.3 is continuously differentiable. Hence $a$ and $v$ satisfy Eqs. (1.1)-(1.6) pointwise.

As an aside we remark that one can rewrite the governing equations in terms of the fiber radius $r$ by setting $r=\sqrt{a}$. The problem then assumes the form

$$
\begin{aligned}
& r_{t}+v r_{z}+\frac{1}{2} v_{z} r=0, \quad t \geq 0, \quad 0 \leq z \leq 1, \\
& \left(r^{2} v_{z}\right)_{z}=0, \\
& r(t, 0)=r_{0}(t), \quad t \geq 0 \\
& r(0, z)=r_{\alpha}(z), \quad 0 \leq z \leq 1 \\
& v(t, 0)=v_{0}(t), \quad v(t, 1)=v_{1}(t), \quad t \geq 0
\end{aligned}
$$

A solution $(r, v)$ of this problem could be obtained in an analogous way as before, thus showing

$$
r \in \bigcap_{k=0}^{2} C^{k}\left(\left[0, t_{0}\right] ; H^{2-k}(0,1)\right)
$$

for some $t_{0}>0$. The regularity of $r$ would imply

$$
a \in \bigcap_{k=0}^{2} C^{k}\left(\left[0, t_{0}\right] ; W^{2-k, 1}(0,1)\right) .
$$

This result would be possibly more appealing since the $L^{1}$-integral of $a$ has the physical meaning of volume and less regularity would be required from $a_{0}$ and $a_{\alpha}$.

\section{A Lagrangian Variable}

Throughout this section we assume that $a=a(t, z)$ is a continuously differentiable, positive solution of Eqs. (1.1)-(1.6) with velocity $v$ on $[0, T) \times[0,1]$ for some $T>0$. We also assume that the boundary data are given on $[0, T]$. Our objective is to find a representation of the fiber cross-sectional area $a$ in terms of a suitably constructed Lagrangian variable and to use this representation to extend $a$ into $t=T$. 
For reference we note first that the velocity $v$ satisfies

$$
v(t, z)=v_{0}(t)+\left(v_{1}(t)-v_{0}(t)\right) \frac{\int_{0}^{z}(a(t, x))^{-1} d x}{\int_{0}^{1}(a(t, x))^{-1} d x} .
$$

Proposition 3.1 The following holds true:

(1) For $0 \leq t<T$ and $0 \leq z \leq 1$

$$
v_{0}(t) \leq v(t, z) \leq v_{1}(t) .
$$

(2) There exists a constant $A>0$ such that for $0 \leq t<T$ and $0 \leq z \leq 1$

$$
0<a(t, z) \leq A \text {. }
$$

The first statement of Proposition 3.1 follows immediately from Eq. (3.1), while the second is readily seen by integration along characteristics.

Next we introduce the Lagrangian variable $Z=Z(t, z)$ by defining

$$
Z(t, z)=\int_{0}^{z} a(t, x) d x-\int_{0}^{t} a_{0}(s) v_{0}(s) d s, \quad 0 \leq t<T, \quad 0 \leq z \leq 1 .
$$

Lemma 3.2 The Lagrangian variable $Z$ has the following properties:

(1) $Z \in C^{1}([0, T) \times[0,1])$

(2) $Z$ satisfies $Z_{t}+v Z_{z}=0$ for $0 \leq t<T, 0 \leq z \leq 1$.

(3) $Z_{z}>0$ and $Z_{t}<0$ for $0 \leq t<T, 0 \leq z \leq 1$.

(4) Image $Z=\left(-\int_{0}^{T} a_{0}(s) v_{0}(s) d s, \int_{0}^{1} a_{\alpha}(x) d x\right]$.

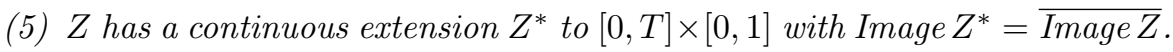

Proof. Statements (1), (2) and (3) are straightforward consequences of the definition of $Z$ and the properties of $a$. Statement (4) follows from (3). For statement (5), we define the extension $Z^{*}$ be setting

$$
Z^{*}(t, z)=\left\{\begin{array}{cl}
Z(t, z) & \text { if } 0 \leq t<T, 0 \leq z \leq 1 \\
\lim _{s}{ }_{T} Z(s, z) & \text { if } t=T, 0 \leq z \leq 1
\end{array}\right.
$$

Clearly, statements (3) and (4) show that the limits are well-defined. Next we note that for $0 \leq s, t<T$ and $0 \leq x, y \leq 1$

$$
\begin{aligned}
Z(t, x)-Z(s, y) & =\int_{t}^{s} a_{0}(\tau) v_{0}(\tau) d \tau+\int_{0}^{x}(a(t, r)-a(s, r)) d r+\int_{y}^{x} a(s, r) d r \\
& =\int_{t}^{s} a_{0}(\tau) v_{0}(\tau) d \tau+\int_{s}^{t} \int_{0}^{x} a_{t}(\tau, r) d r d \tau+\int_{y}^{x} a(s, r) d r \\
& =-\int_{s}^{t} a(\tau, x) v(\tau, x) d \tau+\int_{y}^{x} a(s, r) d r
\end{aligned}
$$


Consequently,

$$
|Z(t, x)-Z(s, y)| \leq \beta|t-s|+\gamma|x-y|
$$

for some constants $\beta, \gamma>0$. As we pass first to the limit $t \nearrow T$ and then to the limit $s \nearrow T$, we see that inequality (3.6) remains valid for $Z^{*}$ rather than $Z$ on $[0, T] \times[0,1]$. The remaining part of statement (5) is obvious.

For later use let us abbreviate

$$
\mathbb{S}=\left(-\int_{0}^{T} a_{0}(s) v_{0}(s) d s, \int_{0}^{1} a_{\alpha}(x) d x\right] .
$$

The salient point for using the Lagrangian variable $Z$ is the following result.

Theorem 3.3 Let $h$ be a continuously differentiable solution of the advection equation

$$
h_{t}+v h_{z}=0
$$

for $0 \leq t<T$ and $0 \leq z \leq 1$. Then there exists a continuously differentiable function $\Phi$ on $\mathbb{S}$ such that

$$
h(t, z)=\Phi(Z(t, z)) \quad \text { for } 0 \leq t<T, 0 \leq z \leq 1 .
$$

Proof. Since $\nabla h \| \nabla Z$, there exists a function $\kappa=\kappa(t, z)$ such that

$$
\nabla h=\kappa \nabla Z .
$$

Clearly, $\kappa$ is continuous on $[0, T) \times[0,1]$. Next, for $c \in \mathbb{S}$, let

$$
\mathbb{D}_{c}=\{(t, z) \in[0, T) \times[0,1] \mid Z(t, z)=c\} .
$$

Suppose $(t(s), z(s))$ is a continuously differentiable curve in $\mathbb{D}_{c}$. Since

$$
Z(t(s), z(s))=c \text { for all } s,
$$

we have

$$
Z_{t} t^{\prime}+Z_{z} z^{\prime}=0
$$

Hence

$$
\frac{d}{d s} h(t(s), z(s))=h_{t} t^{\prime}+h_{z} z^{\prime}=\kappa\left(Z_{t} t^{\prime}+Z_{z} z^{\prime}\right)=0,
$$

thus $h$ is constant on $\mathbb{D}_{c}$. Therefore, setting

$$
\Phi(c)=h(t, z) \quad \text { for }(t, z) \in \mathbb{D}_{c} \text { and } c \in \mathbb{S}
$$

well-defines the map $\Phi$ such that Eq. (3.9) holds true. Next, given $Z_{0}, Z_{1} \in$ $\mathbb{S} \cap[0, \infty)$, we find $0 \leq z_{0}, z_{1} \leq 1$ such that

$$
Z_{0}=Z\left(0, z_{0}\right) \quad \text { and } \quad Z_{1}=Z\left(0, z_{1}\right) .
$$


Hence by the Mean Value Theorem, there is $\xi$ between $z_{0}$ and $z_{1}$ such that

$$
\begin{aligned}
\Phi\left(Z_{1}\right)-\Phi\left(Z_{0}\right) & =h\left(0, z_{1}\right)-h\left(0, z_{0}\right)=\int_{z_{0}}^{z_{1}} h_{z}(0, x) d x \\
& =\int_{z_{0}}^{z_{1}} \kappa(0, x) Z_{z}(0, x) d x=\kappa(0, \xi)\left(Z_{1}-Z_{0}\right) .
\end{aligned}
$$

Since the map $z \mapsto Z(0, z)$ has a continuous inverse, we can define the continuous function $\tilde{\kappa}$ by

$$
\tilde{\kappa}(Z)=\kappa(0, z) \text { for } Z=Z(0, z)
$$

Consequently,

$$
\Phi\left(Z_{1}\right)-\Phi\left(Z_{0}\right)=\tilde{\kappa}(\Xi)\left(Z_{1}-Z_{0}\right)
$$

with $\Xi$ between $Z_{0}$ and $Z_{1}$. This equation shows that $\Phi$ is continuously differentiable on $\mathbb{S} \cap[0, \infty)$ with derivative $\tilde{\kappa}$. In an analogous fashion we obtain the continuous differentiability of $\Phi$ on $\mathbb{S} \cap(-\infty, 0]$. The one-sided derivatives of $\Phi$ at $Z=0$ coincide by continuity of $\kappa$.

Theorem 3.4 The cross-sectional area $a=a(t, z)$ has a representation in the form

$$
a(t, z)=\Phi(Z(t, z))+\Psi(t)
$$

for $0 \leq t<T, 0 \leq z \leq 1$ with $\Phi \in C^{1}(\mathbb{S})$ and $\Psi$ given by

$$
\Psi(t)=-\int_{0}^{t} \frac{v_{1}(s)-v_{0}(s)}{\int_{0}^{1} a(s, x)^{-1} d x} d s
$$

Proof. If we let $\Psi(t)=-\int_{0}^{t} a(s, z) v_{z}(s, z) d s$, then $\Psi$ can written in the form (3.20) by Eq. (1.2). Since $\Psi$ is independent of $z$, we have

$$
(a-\Psi)_{t}+v(a-\Psi)_{z}=0
$$

Consequently, Theorem 3.3 implies the claim.

Corollary 3.5 The following holds true:

(1) The cross-sectional area $a=a(t, z)$ has a continuous extension $a^{*}$ to all of $[0, T] \times[0,1]$.

(2) The extension $Z^{*}$ of the Lagrangian variable is differentiable with respect to $z$ on $[0, T] \times[0,1]$ such that $Z_{z}^{*}=a^{*}$. 
Proof. First we note that the function $\Psi$ has a continuous extension $\Psi^{*}$ to $[0, T]$ since the integrand in Eq. (3.20) is bounded. Then we observe that the image of $(t, z) \mapsto Z^{*}(t, z)$ is contained in $\mathbb{S}$ for $t=T, 0<z \leq 1$. Hence $(t, z) \mapsto \Phi(Z(t, z))$ extends to the continuous function $\Phi\left(Z^{*}\right)$ on $([0, T] \times[0,1]) \backslash(\{T\} \times\{0\})$. The representation (3.19) of $a$ implies then that $a$ has a continuous extension with the exception of the missing point $t=T, z=0$. For this point the extension is, however, given by the boundary data $a_{0}(T)$. Hence statement (1) is established. Statement (2) follows from the definition of $Z$ by Eq. (3.4) and the analogous representation of $Z^{*}$.

\section{A "No Breakup" Result}

In this section we assume again that $a=a(t, z)$ is a continuously differentiable, positive solution of Eqs. (1.1)-(1.6) with velocity $v$ on $[0, T) \times[0,1]$ for some $T>0$ and that the boundary data are given on $[0, T]$. We will use the representation of the cross-sectional area $a$ in the form (3.19) to prove that the fiber radius stays bounded away from 0 over the time interval $[0, T)$. Our argument proceeds by contradiction.

Theorem 4.1 The extension $a^{*}=a^{*}(t, z)$ of the cross-sectional area is strictly positive on $[0, T] \times[0,1]$.

Proof. Suppose there exists $z_{0} \in(0,1]$ such that $a^{*}\left(T, z_{0}\right)=0$. Let $Z_{0}=$ $Z^{*}\left(T, z_{0}\right)$. Since $Z_{0}$ lies in the interior of $\mathbb{S}$, we have

$$
\Phi\left(Z_{0}\right)+\Psi^{*}(T)=0
$$

Let $U$ be a neighborhood of $Z_{0}$ contained in $\mathbb{S}^{o}$. Then there exists a constant $K>0$ such that

$$
\left|\Phi(X)-\Phi\left(Z_{0}\right)\right| \leq K\left|X-Z_{0}\right| \quad \text { for all } X \in U
$$

by continuous differentiability of $\Phi$ on $\mathbb{S}$. Since for $0<z \leq 1$

$$
Z_{z}^{*}(T, z)=a^{*}(T, z)=\Phi\left(Z^{*}(T, z)\right)+\Psi^{*}(T)=\Phi\left(Z^{*}(T, z)\right)-\Phi\left(Z_{0}\right)
$$

by Eq. (4.1), the map $z \mapsto Z^{*}(T, z)$ satisfies the ordinary differential equation

$$
X^{\prime}=\Phi(X)-\Phi\left(Z_{0}\right)
$$

However, the Lipschitz continuity (4.2) of $\Phi$ together with the Picard-Lindelöf Theorem proves that $Z^{*}(T, z)=Z_{0}$ for all $z$ in $\left(z_{0}-\epsilon, z_{0}\right]$ for some sufficiently small $\epsilon>0$. Hence $a^{*}(T, z)=0$ for all $z$ in $\left(z_{0}-\epsilon, z_{0}\right]$. Now choose $t^{\prime} \in[0, T)$ such that

$$
\int_{t^{\prime}}^{T} v_{1}(s) d s<\frac{\epsilon}{2} .
$$


Then for $x \in\left(z_{0}-\epsilon, z_{0}-\frac{\epsilon}{2}\right)$ we define the function $z(t ; x)$ for $t^{\prime} \leq t<T$ by

$$
z_{t}(t ; x)=v(t, z(t ; x)), \quad z\left(t^{\prime} ; x\right)=x .
$$

Observe that for $t^{\prime} \leq t<T$

$$
z\left(t ; z_{1}\right)<z\left(t ; z_{2}\right), \quad \text { if } z_{1}<z_{2}
$$

by uniqueness of solutions. Moreover, $t \mapsto z(t ; x)$ with $x \in\left(z_{0}-\epsilon, z_{0}-\frac{\epsilon}{2}\right)$, given implicitly by

$$
z(t ; x)=x+\int_{t^{\prime}}^{t} v(s, z(s ; x)) d s,
$$

has a continuous extension $z^{*}$ to all of $\left[t^{\prime}, T\right]$ since the integrand is bounded. Since $v_{z}(t, z)>0$ for $0 \leq t<T, 0 \leq z \leq 1$, the monotonicity of $z$ with respect to initial data in (4.7) immediately implies

$$
z^{*}\left(T ; z_{1}\right)<z^{*}\left(T ; z_{2}\right) \text { if } z_{1}<z_{2} .
$$

By continuity, $x \mapsto z^{*}(T ; x)$ maps the interval $\left(z_{0}-\epsilon, z_{0}-\frac{\epsilon}{2}\right)$ to a subinterval in $[0,1]$ (with nonempty interior). Since

$$
z_{0}-\epsilon<z^{*}(T ; x) \leq x+\int_{t^{\prime}}^{T} v_{1}(s) d s<z_{0}-\frac{\epsilon}{2}+\frac{\epsilon}{2}=z_{0},
$$

this subinterval is contained in $\left(z_{0}-\epsilon, z_{0}\right]$. Finally, since by definition of $z(t ; x)$

$$
Z\left(t^{\prime}, x\right)=Z(t, z(t ; x)) \quad \text { for } t^{\prime} \leq t<T, z_{0}-\epsilon<x<z_{0}-\frac{\epsilon}{2}
$$

and by uniqueness of continuous extensions

$$
\lim _{t \nearrow T} Z(t, z(t ; x))=Z^{*}\left(T, z^{*}(T ; x)\right)=Z_{0} \quad \text { for } z_{0}-\epsilon<x<z_{0}-\frac{\epsilon}{2},
$$

we conclude that

$$
Z\left(t^{\prime}, x\right)=Z_{0} \quad \text { for all } z_{0}-\epsilon<x<z_{0}-\frac{\epsilon}{2},
$$

in contradiction to the strict monotonicity of $z \mapsto Z\left(t^{\prime}, z\right)$, given in Lemma 3.2 .

Since the cross-sectional area is bounded below, we can extend the velocity using Eq. (3.1).

Corollary 4.2 The velocity $v=v(t, z)$ has a continuous extension $v^{*}$ to all of $[0, T] \times[0,1]$.

\section{Global Existence}

The results of the previous section allow us to draw some straightforward conclusions. We begin by extending the representation of the cross-sectional area $a$ in terms of the Lagrangian variable to obtain stronger regularity. 
Theorem 5.1 Let $a^{*}=a^{*}(t, z)$ and $v^{*}=v^{*}(t, z)$ be the extensions of the cross-sectional area and velocity on $[0, T] \times[0,1]$, given by Theorem 4.1 and Corollary 4.2, respectively. Then $a^{*}$ has a representation in the form

$$
a^{*}(t, z)=\Phi^{*}\left(Z^{*}(t, z)\right)+\Psi^{*}(t)
$$

for $0 \leq t \leq T, 0 \leq z \leq 1$ with $\Phi^{*} \in C^{1}(\overline{\mathbb{S}})$ and $\Psi^{*} \in C^{1}([0, T])$ given by

$$
\Psi^{*}(t)=-\int_{0}^{t} \frac{v_{1}(s)-v_{0}(s)}{\int_{0}^{1} a^{*}(s, x)^{-1} d x} d s .
$$

Consequently, $a^{*}$ and $v^{*}$ are continuously differentiable for $0 \leq t \leq T, 0 \leq z \leq 1$ and satisfy

$$
a_{t}^{*}+\left(v^{*} a^{*}\right)_{z}=0, \quad\left(a^{*} v_{z}^{*}\right)_{z}=0 \quad \text { on }[0, T] \times[0,1] .
$$

Proof. First we note that $\Psi^{*}$ is well defined and continuously differentiable on $[0, T]$. Next we observe that for $t<T$

$$
\dot{a}_{0}(t)+v_{0}(t) a_{z}(t, 0)+v_{z}(t, 0) a_{0}(t)=0 .
$$

Hence $a_{z}(t, 0)$ can be expressed in terms of the other quantities. Thus we readily see that the $\operatorname{limit} \lim _{t} T_{T} a_{z}(t, 0)$ exists. Since we also have for $\Phi$ given by Theorem 3.4

$$
a_{z}(t, 0)=\Phi^{\prime}(Z(t, 0)) a_{0}(t), \quad 0 \leq t<T
$$

we can define a map $\Omega$ on $\overline{\mathbb{S}}$ by setting

$$
\Omega(X)=\left\{\begin{array}{cl}
\Phi^{\prime}(X) & \text { if } X \in \mathbb{S} \\
\frac{\lim _{t \nearrow T} a_{z}(t, 0)}{a_{0}(T)} & \text { if } X=-\int_{0}^{T} a_{0}(s) v_{0}(s) d s .
\end{array}\right.
$$

It is readily seen that $\Omega$ is well-defined and continuous on $\overline{\mathbb{S}}$. Now we define the extension of $\Phi^{*}$ to $\overline{\mathbb{S}}$ by

$$
\Phi^{*}(X)=\Phi(0)+\int_{0}^{X} \Omega(Y) d Y
$$

Then the representation (5.1) and the regularity of $\Phi^{*}$ are obvious. Consequently, $a^{*}$ and $v^{*}$ are continuously differentiable and satisfy Eqs. (5.3) on $[0, T] \times[0,1]$.

Finally we can state our global existence and uniqueness theorem for the equations of fiber spinning. 
Theorem 5.2 Let $t^{\prime}>0$. Suppose that

$$
\begin{aligned}
& a_{0} \in H^{2}\left(0, t^{\prime}\right), \quad a_{\alpha} \in H^{2}(0,1), \quad a_{0}>0, \quad a_{\alpha}>0, \\
& v_{0}, v_{1} \in C^{1}\left(\left[0, t^{\prime}\right]\right), \quad 0<v_{0}<v_{1}
\end{aligned}
$$

are given such that the compatibility conditions

$$
\begin{aligned}
& a_{0}(0)=a_{\alpha}(0), \\
& \dot{a}_{0}(0)+v_{0} a_{\alpha}^{\prime}(0)+\frac{v_{1}(0)-v_{0}(0)}{\int_{0}^{1} a_{\alpha}^{-1}(x) d x}=0
\end{aligned}
$$

hold. Then the boundary-initial value problem (1.1)-(1.6) has a unique solution $(a, v)$ on $\left[0, t^{\prime}\right] \times[0,1]$ with the properties

$$
\begin{aligned}
& a \in \bigcap_{k=0}^{2} C^{k}\left(\left[0, t^{\prime}\right] ; H^{2-k}(0,1)\right), \quad a>0, \\
& v \in C^{1}\left(\left[0, t^{\prime}\right] ; H^{1}(0,1)\right) \cap C\left(\left[0, t^{\prime}\right] ; H^{2}(0,1)\right), \\
& a, v \in \mathbb{B} \mathbb{R}\left(0, t^{\prime} ; 0,1\right) .
\end{aligned}
$$

Proof. We proceed by showing that the set $\mathbb{T}$ of all times $t_{0} \in\left(0, t^{\prime}\right]$ such that the problem (1.1)-(1.6) has a solution on $\left[0, t_{0}\right] \times[0,1]$ in the sense of Theorem 2.3 is both open and closed in $\left[0, t^{\prime}\right]$.

To this end, suppose $(\tilde{a}, \tilde{v})$ is a local solution on $\left[0, t_{0}\right] \times[0,1]$ for some $0<t_{0}<t^{\prime}$. Since this solution is continuously differentiable and positive such that $\tilde{a}\left(t_{0}, \cdot\right)$ is in $H^{2}(0,1)$, we can apply Theorem 2.3 to find a local-in-time solution $(\hat{a}, \hat{v})$ of the problem

$$
\begin{aligned}
& a_{t}+(a v)_{z}=0, \quad t \geq t_{0}, \quad 0 \leq z \leq 1, \\
& \left(a v_{z}\right)_{z}=0, \\
& a(t, 0)=a_{0}(t), \quad t \geq t_{0}, \\
& v(t, 0)=v_{0}(t), \quad v(t, 1)=v_{1}(t), \quad t \geq t_{0} \\
& a\left(t_{0}, z\right)=\tilde{a}\left(t_{0}, z\right), \quad 0 \leq z \leq 1 .
\end{aligned}
$$

Observe that the conditions of Theorem 2.3 hold true (modulo a shift from $t=0$ to $\left.t=t_{0}\right)$. Hence there is $\epsilon>0$ such that this problem has the solution $(\hat{a}, \hat{v})$ on $\left[t_{0}, t_{0}+\epsilon\right] \times[0,1]$. When we define $(a, v)$ piecewise to equal $(\tilde{a}, \tilde{v})$ on $\left[0, t_{0}\right] \times[0,1]$ and $(\hat{a}, \hat{v})$ on $\left[t_{0}, t_{0}+\epsilon\right] \times[0,1],(a, v)$ is a solution in the sense of Theorem 2.3 on $\left[0, t_{0}+\epsilon\right] \times[0,1]$. To see this, note that the required regularity of $a$ and $v$ follows immediately from the regularity of $\tilde{a}, \hat{a}, \tilde{v}, \hat{v}$ and the way how partial derivatives are related via Eq. (1.1). Hence $\mathbb{T}$ is open.

Next let $t^{*}=\sup \mathbb{T}$. Then there is $(a, v)$ which solves problem (1.1)-(1.6) in the sense of Theorem 2.3 on $\left[0, t_{0}\right] \times[0,1]$ for any $t_{0}<t^{*}$. Hence $(a, v)$ is continuously differentiable on $\left[0, t^{*}\right) \times[0,1]$. In light of Theorem 4.1 and 
Theorem $5.1 a$ and $v$ extend to continuously differentiable functions $a^{*}$ and $v^{*}$ on $\left[0, t^{*}\right] \times[0,1]$ such that $a^{*}>0$. Eq. (3.1) shows for $a$ and $v$ replaced by $a^{*}$ and $v^{*}$ that $v^{*}$ and $v_{z}^{*} a^{*}$ belong to $\mathbb{B R}\left(0, t^{*} ; 0,1\right)$. Consequently, Lemma 2.2 and Theorem 5.1 give that $\left(a^{*}, v^{*}\right)$ is a solution of problem (1.1)-(1.6) in the sense of Theorem 2.3 on $\left[0, t^{*}\right] \times[0,1]$. Hence $\mathbb{T}$ is closed.

\section{Conclusion}

The problem discussed in this work sheds light on an important asymptotic regime of the Stokes equations with free boundary. The pivotal point in our approach was the characterization of the fiber cross-sectional area by a Lagrangian variable. This characterization was used to prove a no-breakup result for viscous fibers and to extend the cross-sectional area to a continuously differentiable function on the closed time-space domain. These two results allowed us to extend a local-in-time solution of the governing equations to a global-intime solution. The Lagrangian variable technique is of interest in its own right. This technique is likely to be applicable to a broader class of problems and has therefore been studied thoroughly here.

In this work breakup or failure of a filament is understood in the mathematical sense that the fiber cross-sectional area of the continuum mechanical model stays bounded away from 0 over finite time intervals. Clearly, this condition is not appropriate to describe the failure or non-failure of real-world fibers and must therefore be distinguished from actual breakup of filaments. The no-breakup result given here might appear surprising at first glance. However, Hassager, Kolte and Renardy [7] made a similar observation when a viscous fluid filament is stretched on both ends. Related results on the thin filament approximation of highly viscous fluids suggest, however, that the situation changes completely when surface tension is accounted for as shown by Renardy in $[10,11]$. Since surface tension driven instabilities can lead to breakup, filament failure should also be expected for the equations of fiber spinning when surface tension is included in the governing equations.

Acknowledgment. The authors acknowledge financial support through NSF-Grants DMS 0709197 (TH) and DMS 1008426 (MR). They also express their gratitude to their colleagues at the Institute for Mathematics and its Applications at the University of Minnesota for their hospitality, while the authors were in residence in fall 2009. Central parts of this work were written during this stay.

\section{References}

[1] T. Hagen, Elongational Flows in Polymer Processing, Doctoral Dissertation, Virginia Polytechnic Institute and State University, 1998. 
[2] T. Hagen, On viscoelastic fluids in elongation. In Advances in Mathematics Research, vol. 1, G. Oyibo, ed., Nova Science, New York 2002.

[3] T. Hagen, Jeffreys fluids in forced elongation, J. Math. Anal. Appl. 288 (2003), 634-645.

[4] T. Hagen and D. Kurmashev, Analysis of fiber spinning for the upperconvected Maxwell fluid, submitted.

[5] T. Hagen and M. Renardy, On the equations of fiber spinning in nonisothermal viscous flow. In Topics in Nonlinear Analysis. The Herbert Amann Anniversary Volume, J. Escher and G. Simonett, eds., Birkhäuser, Basel 1999.

[6] T. Hagen and M. Renardy, Non-adiabatic elongational flows of viscoelastic melts, Z. Angew. Math. Phys. 51 (2000), 845-866.

[7] O. Hassager, M. I. Kolte and M. Renardy, Failure and nonfailure of fluid filaments in extension, J. Non-Newtonian Fluid Mech. 76 (1998), 137-152.

[8] S. Kase and T. Matsuo, Studies on melt spinning. I. Fundamental equations on the dynamics of melt spinning, J. Appl. Polym. Sci. A. 3 (1965), 25412554 .

[9] M. A. Matovich and J. R. A. Pearson, Spinning a molten threadline steady-state isothermal viscous flows, Ind. Eng. Chem. Fundam. 8 (1969), $512-520$.

[10] M. Renardy, Finite time breakup of viscous filaments, Z. Angew. Math. Physik 52 (2001), 881-887.

[11] M. Renardy, A comment on self-similar breakup for inertialess Newtonian liquid jets, IMA J. Applied Math. 70 (2005), 353-358. 